\title{
MultiFC: A Real-World Multi-Domain Dataset for Evidence-Based Fact Checking of Claims
}

\author{
Isabelle Augenstein Christina Lioma Dongsheng Wang Lucas Chaves Lima \\ Casper Hansen Christian Hansen Jakob Grue Simonsen \\ Department of Computer Science \\ University of Copenhagen \\ \{augenstein, c.lioma, wang, lcl, c.hansen, chrh, simonsen\}@di.ku.dk
}

\begin{abstract}
We contribute the largest publicly available dataset of naturally occurring factual claims for the purpose of automatic claim verification. It is collected from 26 fact checking websites in English, paired with textual sources and rich metadata, and labelled for veracity by human expert journalists. We present an in-depth analysis of the dataset, highlighting characteristics and challenges. Further, we present results for automatic veracity prediction, both with established baselines and with a novel method for joint ranking of evidence pages and predicting veracity that outperforms all baselines. Significant performance increases are achieved by encoding evidence, and by modelling metadata. Our best-performing model achieves a Macro F1 of $49.2 \%$, showing that this is a challenging testbed for claim veracity prediction.
\end{abstract}

\section{Introduction}

Misinformation and disinformation are two of the most pertinent and difficult challenges of the information age, exacerbated by the popularity of social media. In an effort to counter this, a significant amount of manual labour has been invested in fact checking claims, often collecting the results of these manual checks on fact checking portals or websites such as politifact.com or snopes.com. In a parallel development, researchers have recently started to view fact checking as a task that can be partially automated, using machine learning and NLP to automatically predict the veracity of claims. However, existing efforts either use small datasets consisting of naturally occurring claims (e.g. Mihalcea and Strapparava (2009); Zubiaga et al. (2016)), or datasets consisting of artificially constructed claims such as FEVER (Thorne et al., 2018). While the latter offer valuable contributions to further automatic claim verification work, they cannot replace real-world datasets.

\begin{tabular}{|c|c|}
\hline Feature & Value \\
\hline ClaimID & farg-00004 \\
\hline Claim & $\begin{array}{l}\text { Mexico and Canada assemble } \\
\text { cars with foreign parts and send } \\
\text { them to the U.S. with no tax. }\end{array}$ \\
\hline Label & distorts \\
\hline Claim URL & $\begin{array}{l}\text { https://www.factcheck.org/2018/ } \\
\text { 10/factchecking-trump-on-trade/ }\end{array}$ \\
\hline Reason & None \\
\hline Category & the-factcheck-wire \\
\hline Speaker & Donald Trump \\
\hline Checker & Eugene Kiely \\
\hline Tags & $\begin{array}{l}\text { North American Free Trade } \\
\text { Agreement }\end{array}$ \\
\hline Claim Entities & United_States, Canada, Mexico \\
\hline Article Title & FactChecking Trump on Trade \\
\hline Publish Date & October 3,2018 \\
\hline Claim Date & Monday, October 1, 2018 \\
\hline
\end{tabular}

Table 1: An example of a claim instance. Entities are obtained via entity linking. Article and outlink texts, evidence search snippets and pages are not shown.

Contributions. We introduce the currently largest claim verification dataset of naturally occurring claims. ${ }^{1}$ It consists of 34,918 claims, collected from 26 fact checking websites in English; evidence pages to verify the claims; the context in which they occurred; and rich metadata (see Table 1 for an example). We perform a thorough analysis to identify characteristics of the dataset such as entities mentioned in claims. We demonstrate the utility of the dataset by training state of the art veracity prediction models, and find that evidence pages as well as metadata significantly contribute to model performance. Finally, we propose a novel model that jointly ranks evidence pages and performs veracity prediction. The best-performing model achieves a Macro F1 of $49.2 \%$, showing that this is a non-trivial dataset with remaining challenges for future work.

\footnotetext{
${ }^{1}$ The dataset is found here: https://copenlu. github.io/publication/2019_emnlp_ augenstein/
} 


\section{Related Work}

\subsection{Datasets}

Over the past few years, a variety of mostly small datasets related to fact checking have been released. An overview over core datasets is given in Table 2. The datasets can be grouped into four categories (I-IV). Category I contains datasets aimed at testing how well the veracity ${ }^{3}$ of a claim can be predicted using the claim alone, without context or evidence documents. Category II contains datasets bundled with documents related to each claim - either topically related to provide context, or serving as evidence. Those documents are, however, not annotated. Category III is for predicting veracity; they encourage retrieving evidence documents as part of their task description, but do not distribute them. Finally, category IV comprises datasets annotated for both veracity and stance. Thus, every document is annotated with a label indicating whether the document supports or denies the claim, or is unrelated to it. Additional labels can then be added to the datasets to better predict veracity, for instance by jointly training stance and veracity prediction models.

Methods not shown in the table, but related to fact checking, are stance detection for claims (Ferreira and Vlachos, 2016; Pomerleau and Rao, 2017; Augenstein et al., 2016a; Kochkina et al., 2017; Augenstein et al., 2016b; Zubiaga et al., 2018; Riedel et al., 2017), satire detection (Rubin et al., 2016), clickbait detection (Karadzhov et al., 2017), conspiracy news detection (Tacchini et al., 2017), rumour cascade detection (Vosoughi et al., 2018) and claim perspectives detection (Chen et al., 2019).

Claims are obtained from a variety of sources, including Wikipedia, Twitter, criminal reports and fact checking websites such as politifact.com and snopes.com. The same goes for documents - these are often websites obtained through Web search queries, or Wikipedia documents, tweets or Facebook posts. Most datasets contain a fairly small number of claims, and those that do not, often lack evidence documents. An exception is Thorne et al. (2018), who create a Wikipedia-based fact checking dataset. While a good testbed for developing deep neural architectures, their dataset is artificially constructed and can thus not take metadata

\footnotetext{
${ }^{3}$ We use veracity, claim credibility, and fake news prediction interchangeably here - these terms are often conflated in the literature and meant to have the same meaning.
}

about claims into account.

Contributions: We provide a dataset that, uniquely among extant datasets, contains a large number of naturally occurring claims and rich additional meta-information.

\subsection{Methods}

Fact checking methods partly depend on the type of dataset used. Methods only taking into account claims typically encode those with CNNs or RNNs (Wang, 2017; Pérez-Rosas et al., 2018), and potentially encode metadata (Wang, 2017) in a similar way. Methods for small datasets often use handcrafted features that are a mix of bag of word and other lexical features, e.g. LIWC, and then use those as input to a SVM or MLP (Mihalcea and Strapparava, 2009; Pérez-Rosas et al., 2018; Baly et al., 2018). Some use additional Twitter-specific features (Enayet and El-Beltagy, 2017). More involved methods taking into account evidence documents, often trained on larger datasets, consist of evidence identification and ranking following a neural model that measures the compatibility between claim and evidence (Thorne et al., 2018; Mihaylova et al., 2018; Yin and Roth, 2018).

Contributions: The latter category above is the most related to our paper as we consider evidence documents. However, existing models are not trained jointly for evidence identification, or for stance and veracity prediction, but rather employ a pipeline approach. Here, we show that a joint approach that learns to weigh evidence pages by their importance for veracity prediction can improve downstream veracity prediction performance.

\section{Dataset Construction}

We crawled a total of 43,837 claims with their metadata (see details in Table 11). We present the data collection in terms of selecting sources, crawling claims and associated metadata (Section 3.1); retrieving evidence pages; and linking entities in the crawled claims (Section 3.3).

\subsection{Selection of sources}

We crawled all active fact checking websites in English listed by Duke Reporters' $\mathrm{Lab}^{4}$ and on the Fact Checking Wikipedia page. ${ }^{5}$ This resulted in

\footnotetext{
${ }^{4}$ https://reporterslab.org/ fact-checking/

${ }^{5}$ https://en.wikipedia.org/wiki/Fact_ checking
} 


\begin{tabular}{lcccc}
\hline Dataset & \# Claims & Labels & metadata & Claim Sources \\
\hline I: Veracity prediction w/o evidence & 12,836 & 6 & Yes & Politifact \\
Wang (2017) & 980 & 2 & No & News Websites \\
Pérez-Rosas et al. (2018) & & & & \\
\hline II: Veracity & 275 & 2 & No & Criminal Reports \\
Bachenko et al. (2008) & 600 & 2 & No & Crowd Authors \\
Mihalcea and Strapparava (2009) & 1,049 & 5 & No & Twitter \\
Mitra and Gilbert (2015) $\dagger$ & 10,000 & 2 & No & Google, Wikipedia \\
Ciampaglia et al. (2015) $\dagger$ & 5,013 & 2 & Yes & Wikipedia, Snopes \\
Popat et al. (2016) & 23,921 & 2 & Yes & Politifact, gossipcop.com \\
Shu et al. (2018) $\dagger$ & 10,564 & $2-6$ & Yes & Fact Checking Websites \\
Datacommons Fact Check ${ }^{2}$ & 150 & 3 & No & factcheck.org, Snopes \\
\hline III: Veracity (evidence encouraged, but not provided) & & & \\
Barrn-Cedeo et al. (2018) & & & Yes & Politifact, Channel 4 News \\
\hline IV: Veracity + stance & 106 & 5 & Yes & Twitter \\
Vlachos and Riedel (2014) & 330 & 3 & Yes & Twitter \\
Zubiaga et al. (2016) & 325 & 3 & No & ara.reuters.com, verify-sy.com \\
Derczynski et al. (2017) & 422 & 2 & No & Wikipedia \\
Baly et al. (2018) & 185,445 & 3 & & \\
Thorne et al. (2018) $\dagger$ & & & Yes & Fact Checking Websites \\
\hline \hline V: Veracity + evidence relevancy & 36,534 & $2-40$ &
\end{tabular}

Table 2: Comparison of fact checking datasets. $\dagger$ indicates claims are not 'naturally occuring': Mitra and Gilbert (2015) use events as claims; Ciampaglia et al. (2015) use DBPedia tiples as claims; Shu et al. (2018) use tweets as claims; and Thorne et al. (2018) rewrite sentences in Wikipedia as claims.

38 websites in total (shown in Table 11). Out of these, ten websites could not be crawled, as further detailed in Table 9. In the later experimental descriptions, we refer to the part of the dataset crawled from a specific fact checking website as a domain, and we refer to each website as source.

From each source, we crawled the ID, claim, label, URL, reason for label, categories, person making the claim (speaker), person fact checking the claim (checker), tags, article title, publication date, claim date, as well as the full text that appears when the claim is clicked. Lastly, the above full text contains hyperlinks, so we further crawled the full text that appears when each of those hyperlinks are clicked (outlinks).

There were a number of crawling issues, e.g. security protection of websites with SSL/TLS protocols, time out, URLs that pointed to pdf files instead of HTML content, or unresolvable encoding. In all of these cases, the content could not be retrieved. For some websites, no veracity labels were available, in which case, they were not selected as domains for training a veracity prediction model. Moreover, not all types of metadata (category, speaker, checker, tags, claim date, publish date) were available for all websites; and availability of articles and full texts differs as well.

We performed semi-automatic cleansing of the dataset as follows. First, we double-checked that the veracity labels would not appear in claims. For some domains, the first or last sentence of the claim would sometimes contain the veracity label, in which case we would discard either the full sentence or part of the sentence. Next, we checked the dataset for duplicate claims. We found 202 such instances, 69 of them with different labels. Upon manual inspection, this was mainly due to them appearing on different websites, with labels not differing much in practice (e.g. 'Not true', vs. 'Mostly False'). We made sure that all such duplicate claims would be in the training split of the dataset, so that the models would not have an unfair advantage. Finally, we performed some minor manual merging of label types for the same domain where it was clear that they were supposed to denote the same level of veracity (e.g. 'distorts', 'distorts the facts').

This resulted in a total of 36,534 claims with their metadata. For the purposes of fact verification, we discarded instances with labels that occur fewer than 5 times, resulting in 34,918 claims. The number of instances, as well as labels per domain, are shown in Table 6 and label names in Table 10 in the appendix. The dataset is split into a training part (80\%) and a development and testing part (10\% each) in a label-stratified manner. Note that 
the domains vary in the number of labels, ranging from 2 to 27. Labels include both straight-forward ratings of veracity ('correct', 'incorrect'), but also labels that would be more difficult to map onto a veracity scale (e.g. 'grass roots movement!', 'misattributed', 'not the whole story'). We therefore do not postprocess label types across domains to map them onto the same scale, and rather treat them as is. In the methodology section (Section 4), we show how a model can be trained on this dataset regardless by framing this multi-domain veracity prediction task as a multi-task learning (MTL) one.

\subsection{Retrieving Evidence Pages}

The text of each claim is submitted verbatim as a query to the Google Search API (without quotes). The 10 most highly ranked search results are retrieved, for each of which we save the title; Google search rank; URL; time stamp of last update; search snippet; as well as the full Web page. We acknowledge that search results change over time, which might have an effect on veracity prediction. However, studying such temporal effects is outside the scope of this paper. Similar to Web crawling claims, as described in Section 3.1, the corresponding Web pages can in some cases not be retrieved, in which case fewer than 10 evidence pages are available. The resulting evidence pages are from a wide variety of URL domains, though with a predictable skew towards popular websites, such as Wikipedia or The Guardian (see Table 3 for detailed statistics).

\subsection{Entity Detection and Linking}

To better understand what claims are about, we conduct entity linking for all claims. Specifically, mentions of people, places, organisations, and other named entities within a claim are recognised and linked to their respective Wikipedia pages, if available. Where there are different entities with the same name, they are disambiguated. For this, we apply the state-of-the-art neural entity linking model by Kolitsas et al. (2018). This results in a total of 25,763 entities detected and linked to Wikipedia, with a total of 15,351 claims involved, meaning that $42 \%$ of all claims contain entities that can be linked to Wikipedia. Later on, we use entities as additional metadata (see Section 4.3). The distribution of claim numbers according to the number of entities they contain is shown in Figure 1. We observe that the majority of claims have

\begin{tabular}{ll}
\hline Domain & $\%$ \\
\hline https://en.wikipedia.org/ & 4.425 \\
https://www.snopes.com/ & 3.992 \\
https://www.washingtonpost.com/ & 3.025 \\
https://www.nytimes.com/ & 2.478 \\
https://www.theguardian.com/ & 1.807 \\
https://www.youtube.com/ & 1.712 \\
https://www.dailymail.co.uk/ & 1.558 \\
https://www.usatoday.com/ & 1.279 \\
https://www.politico.com/ & 1.241 \\
http://www.politifact.com/ & 1.231 \\
https://www.pinterest.com/ & 1.169 \\
https://www.factcheck.org/ & 1.09 \\
https://www.gossipcop.com/ & 1.073 \\
https://www.cnn.com/ & 1.065 \\
https://www.npr.org/ & 0.957 \\
https://www.forbes.com/ & 0.911 \\
https://www.vox.com/ & 0.89 \\
https://www.theatlantic.com/ & 0.88 \\
https://twitter.com/ & 0.767 \\
https://www.hoax-slayer.net/ & 0.655 \\
http://time.com/ & 0.554 \\
https://www.bbc.com/ & 0.551 \\
https://www.nbcnews.com/ & 0.515 \\
https://www.cnbc.com/ & 0.514 \\
https://www.cbsnews.com/ & 0.503 \\
https://www.facebook.com/ & 0.5 \\
https://www.newyorker.com/ & 0.495 \\
https://www.foxnews.com/ & 0.468 \\
https://people.com/ & 0.439 \\
http://www.cnn.com/ & 0.419 \\
\hline
\end{tabular}

Table 3: The top 30 most frequently occurring URL domains.

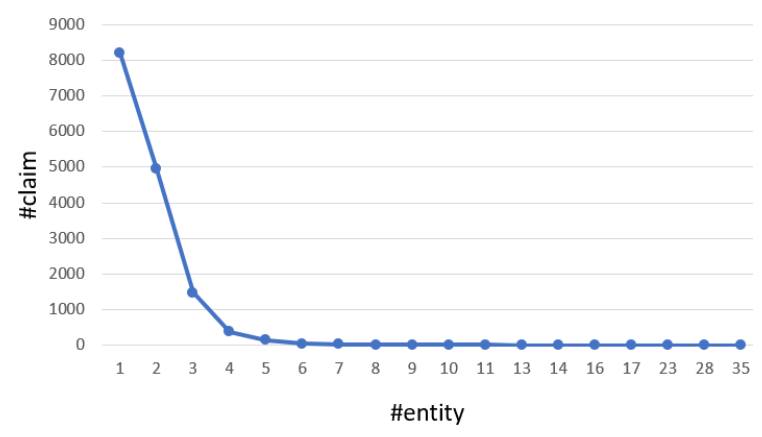

Figure 1: Distribution of entities in claims.

one to four entities, and the maximum number of 35 entities occurs in one claim only. Out of the 25,763 entities, 2,767 are unique entities. The top 30 most frequent entities are listed in Table 4. This clearly shows that most of the claims involve entities related to the United States, which is to be expected, as most of the fact checking websites are US-based.

\section{Claim Veracity Prediction}

We train several models to predict the veracity of claims. Those fall into two categories: those that 


\begin{tabular}{lr}
\hline Entity & Frequency \\
\hline United_States & 2810 \\
Barack_Obama & 1598 \\
Republican_Party_(United_States) & 783 \\
Texas & 665 \\
Democratic_Party_(United_States) & 560 \\
Donald_Trump & 556 \\
Wisconsin & 471 \\
United_States_Congress & 354 \\
Hillary_Rodham_Clinton & 306 \\
Bill_Clinton & 292 \\
California & 285 \\
Russia & 275 \\
Ohio & 239 \\
China & 229 \\
George_W._Bush & 208 \\
Medicare_(United_States) & 206 \\
Australia & 186 \\
Iran & 183 \\
Brad_Pitt & 180 \\
Islam & 178 \\
Iraq & 176 \\
Canada & 174 \\
White_House & 166 \\
New_York_City & 164 \\
Washington,_D.C. & 164 \\
Jennifer_Aniston & 163 \\
Mexico & 158 \\
Ted_Cruz & 152 \\
Federal_Bureau_of_Investigation & 146 \\
Syria & 130 \\
\hline
\end{tabular}

Table 4: Top 30 most frequent entities listed by their Wikipedia URL with prefix omitted

only consider the claims themselves, and those that encode evidence pages as well. In addition, claim metadata (speaker, checker, linked entities) is optionally encoded for both categories of models, and ablation studies with and without that metadata are shown. We first describe the base model used in Section 4.1, followed by introducing our novel evidence ranking and veracity prediction model in Section 4.2, and lastly the metadata encoding model in Section 4.3.

\subsection{Multi-Domain Claim Veracity Prediction with Disparate Label Spaces}

Since not all fact checking websites use the same claim labels (see Table 6, and Table 10 in the appendix), training a claim veracity prediction model is not entirely straight-forward. One option would be to manually map those labels onto one another. However, since the sheer number of labels is rather large (165), and it is not always clear from the guidelines on fact checking websites how they can be mapped onto one another, we opt to learn how these labels relate to one another as part of the veracity prediction model. To do so, we employ the multi-task learning (MTL) approach inspired by collaborative filtering presented in Augenstein et al. (2018) (MTL with LEL-multitask learning with label embedding layer) that excels on pairwise sequence classification tasks with disparate label spaces. More concretely, each domain is modelled as its own task in a MTL architecture, and labels are projected into a fixed-length label embedding space. Predictions are then made by taking the dot product between the claim-evidence embeddings and the label embeddings. By doing so, the model implicitly learns how semantically close the labels are to one another, and can benefit from this knowledge when making predictions for individual tasks, which on their own might only have a small number of instances. When making predictions for individual domains/tasks, both at training and at test time, as well as when calculating the loss, a mask is applied such that the valid and invalid labels for that task are restricted to the set of known task labels.

Note that the setting here slightly differs from Augenstein et al. (2018). There, tasks are less strongly related to one another; for example, they consider stance detection, aspect-based sentiment analysis and natural language inference. Here, we have different domains, as opposed to conceptually different tasks, but use their framework, as we have the same underlying problem of disparate label spaces. A more formal problem definition follows next, as our evidence ranking and veracity prediction model in Section 4.2 then builds on it.

\subsubsection{Problem Definition}

We frame our problem as a multi-task learning one, where access to labelled datasets for $T$ tasks $\mathcal{T}_{1}, \ldots, \mathcal{T}_{T}$ is given at training time with a target task $\mathcal{T}_{T}$ that is of particular interest. The training dataset for task $\mathcal{T}_{i}$ consists of $N$ examples $X_{\mathcal{T}_{i}}=\left\{x_{1}^{\mathcal{T}_{i}}, \ldots, x_{N}^{\mathcal{T}_{i}}\right\}$ and their labels $Y_{\mathcal{T}_{i}}=$ $\left\{\mathbf{y}_{1}^{\mathcal{T}_{i}}, \ldots, \mathbf{y}_{N}^{\mathcal{T}_{i}}\right\}$. The base model is a classic deep neural network MTL model (Caruana, 1993) that shares its parameters across tasks and has taskspecific softmax output layers that output a probability distribution $\mathbf{p}^{\mathcal{T}_{i}}$ for task $\mathcal{T}_{i}$ :

$$
\mathbf{p}^{\mathcal{T}_{i}}=\operatorname{softmax}\left(\mathbf{W}^{\mathcal{T}_{i}} \mathbf{h}+\mathbf{b}^{\mathcal{T}_{i}}\right)
$$

where $\operatorname{softmax}(\mathbf{x})=e^{\mathbf{x}} / \sum_{i=1}^{\|\mathbf{x}\|} e^{\mathbf{x}_{i}}, \mathbf{W}^{\mathcal{T}_{i}} \in$ $\mathbb{R}^{L_{i} \times h}, \mathbf{b}^{\mathcal{T}_{i}} \in \mathbb{R}^{L_{i}}$ is the weight matrix and bias term of the output layer of task $\mathcal{T}_{i}$ respectively, $\mathbf{h} \in \mathbb{R}^{h}$ is the jointly learned hidden rep- 
resentation, $L_{i}$ is the number of labels for task $\mathcal{T}_{i}$, and $h$ is the dimensionality of $\mathbf{h}$. The MTL model is trained to minimise the sum of individual task losses $\mathcal{L}_{1}+\ldots+\mathcal{L}_{T}$ using a negative loglikelihood objective.

Label Embedding Layer. To learn the relationships between labels, a Label Embedding Layer (LEL) embeds labels of all tasks in a joint Euclidian space. Instead of training separate softmax output layers as above, a label compatibility function $c(\cdot, \cdot)$ measures how similar a label with embedding $\mathbf{l}$ is to the hidden representation $\mathbf{h}$ :

$$
c(\mathbf{l}, \mathbf{h})=\mathbf{l} \cdot \mathbf{h}
$$

where $\cdot$ is the dot product. Padding is applied such that $l$ and $h$ have the same dimensionality. Matrix multiplication and softmax are used for making predictions:

$$
\mathbf{p}=\operatorname{softmax}(\mathbf{L h})
$$

where $\mathbf{L} \in \mathbb{R}^{\left(\sum_{i} L_{i}\right) \times l}$ is the label embedding matrix for all tasks and $l$ is the dimensionality of the label embeddings. We apply a task-specific mask to $\mathbf{L}$ in order to obtain a task-specific probability distribution $\mathbf{p}^{\mathcal{T}_{i}}$. The LEL is shared across all tasks, which allows the model to learn the relationships between labels in the joint embedding space.

\subsection{Joint Evidence Ranking and Claim Veracity Prediction}

So far, we have ignored the issue of how to obtain claim representation, as the base model described in the previous section is agnostic to how instances are encoded. A very simple approach, which we report as a baseline, is to encode claim texts only. Such a model ignores evidence for and against a claim, and ends up guessing the veracity based on surface patterns observed in the claim texts.

We next introduce two variants of evidencebased veracity prediction models that encode 10 pieces of evidence in addition to the claim. Here, we opt to encode search snippets as opposed to whole retrieved pages. While the latter would also be possible, it comes with a number of additional challenges, such as encoding large documents, parsing tables or PDF files, and encoding images or videos on these pages, which we leave to future work. Search snippets also have the benefit that they already contain summaries of the part of the page content that is most related to the claim.

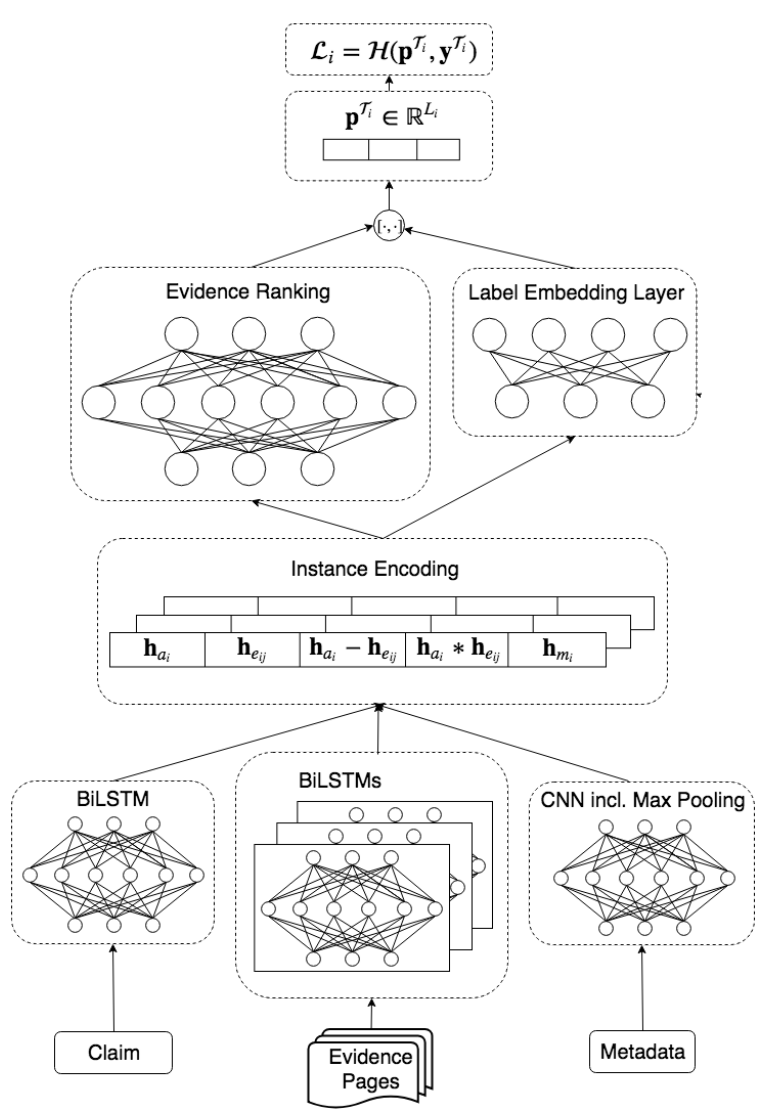

Figure 2: The Joint Veracity Prediction and Evidence Ranking model, shown for one task.

\subsubsection{Problem Definition}

Our problem is to obtain encodings for $N$ examples $X_{\mathcal{T}_{i}}=\left\{x_{1}^{\mathcal{T}_{i}}, \ldots, x_{N}^{\mathcal{T}_{i}}\right\}$. For simplicity, we will henceforth drop the task superscript and refer to instances as $X=\left\{x_{1}, \ldots, x_{N}\right\}$, as instance encodings are learned in a task-agnostic fashion. Each example further consists of a claim $a_{i}$ and $k=10$ evidence pages $E_{k}=\left\{e_{1_{0}}, \ldots, e_{N_{10}}\right\}$.

Each claim and evidence page is encoded with a BiLSTM to obtain a sentence embedding, which is the concatenation of the last state of the forward and backward reading of the sentence, i.e. $\mathbf{h}=$ $\operatorname{BiLSTM}(\cdot)$, where $\mathbf{h}$ is the sentence embedding.

Next, we want to combine claims and evidence sentence embeddings into joint instance representations. In the simplest case, referred to as model variant crawled_avg, we mean average the BiLSTM sentence embeddings of all evidence pages (signified by the overline) and concatenate those with the claim embeddings, i.e.

$$
\mathbf{s}_{g_{i}}=\left[\mathbf{h}_{a_{i}} ; \overline{\mathbf{h}_{E_{i}}}\right]
$$

where $s_{g_{i}}$ is the resulting encoding for training example $i$ and $[\because ; \cdot]$ denotes vector concatenation. 
However, this has the disadvantage that all evidence pages are considered equal.

Evidence Ranking The here proposed alternative instance encoding model, crawled_ranked, which achieves the highest overall performance as discussed in Section 5, learns the compatibility between an instance's claim and each evidence page. It ranks evidence pages by their utility for the veracity prediction task, and then uses the resulting ranking to obtain a weighted combination of all claim-evidence pairs. No direct labels are available to learn the ranking of individual documents, only for the veracity of the associated claim, so the model has to learn evidence ranks implicitly.

To combine claim and evidence representations, we use the matching model proposed for the task of natural language inference by Mou et al. (2016) and adapt it to combine an instance's claim representation with each evidence representation, i.e.

$$
s_{r_{i_{j}}}=\left[\mathbf{h}_{a_{i}} ; \mathbf{h}_{e_{i_{j}}} ; \mathbf{h}_{a_{i}}-\mathbf{h}_{e_{i_{j}}} ; \mathbf{h}_{a_{i}} \cdot \mathbf{h}_{e_{i_{j}}}\right]
$$

where $s_{r_{i_{j}}}$ is the resulting encoding for training example $i$ and evidence page $j,[\cdot ; \cdot]$ denotes vector concatenation, and $\cdot$ denotes the dot product.

All joint claim-evidence representations $\mathbf{s}_{r_{i_{0}}}, \ldots, \mathbf{s}_{r_{i_{10}}}$ are then projected into the binary space via a fully connected layer FC, followed by a non-linear activation function $f$, to obtain a soft ranking of claim-evidence pairs, in practice a 10-dimensional vector,

$$
\mathbf{o}_{i}=\left[f\left(\mathrm{FC}\left(s_{r_{i_{0}}}\right)\right) ; \ldots ; f\left(\mathrm{FC}\left(s_{r_{i_{10}}}\right)\right)\right]
$$

where $[\cdot ; \cdot]$ denotes concatenation.

Scores for all labels are obtained as per (6) above, with the same input instance embeddings as for the evidence ranker, i.e. $s_{r_{i_{j}}}$. Final predictions for all claim-evidence pairs are then obtained by taking the dot product between the label scores and binary evidence ranking scores, i.e.

$$
\mathbf{p}_{i}=\operatorname{softmax}\left(c\left(\mathbf{l}, \mathbf{s}_{\mathbf{r}_{\mathbf{i}}}\right) \cdot \mathbf{o}_{i}\right)
$$

Note that the novelty here is that, unlike for the model described in Mou et al. (2016), we have no direct labels for learning weights for this matching model. Rather, our model has to implicitly learn these weights for each claim-evidence pair in an end-to-end fashion given the veracity labels.

\begin{tabular}{lcc}
\hline Model & Micro F1 & Macro F1 \\
\hline claim-only & 0.469 & 0.253 \\
claim-only_embavg & 0.384 & 0.302 \\
crawled-docavg & 0.438 & 0.248 \\
crawled_ranked & 0.613 & 0.441 \\
\hline claim-only + meta & 0.494 & 0.324 \\
claim-only_embavg + meta & 0.418 & 0.333 \\
crawled-docavg + meta & 0.483 & 0.286 \\
crawled_ranked + meta & $\mathbf{0 . 6 2 5}$ & $\mathbf{0 . 4 9 2}$ \\
\hline
\end{tabular}

Table 5: Results with different model variants on the test set, 'meta' means all metadata is used.

\subsection{Metadata}

We experiment with how useful claim metadata is, and encode the following as one-hot vectors: speaker, category, tags and linked entities. We do not encode 'Reason' as it gives away the label, and do not include 'Checker' as there are too many unique checkers for this information to be relevant. The claim publication date is potentially relevant, but it does not make sense to merely model this as a one-hot feature, so we leave incorporating temporal information to future work. Since all metadata consists of individual words and phrases, a sequence encoder is not necessary, and we opt for a CNN followed by a max pooling operation as used in Wang (2017) to encode metadata for fact checking. The max-pooled metadata representations, denoted $h_{m}$, are then concatenated with the instance representations, e.g. for the most elaborate model, crawled_ranked, these would be concatenated with $s_{c r_{i j}}$.

\section{Experiments}

\subsection{Experimental Setup}

The base sentence embedding model is a BiLSTM over all words in the respective sequences with randomly initialised word embeddings, following Augenstein et al. (2018). We opt for this strong baseline sentence encoding model, as opposed to engineering sentence embeddings that work particularly well for this dataset, to showcase the dataset. We would expect pre-trained contextual encoding models, e.g. ELMO (Peters et al., 2018), ULMFit (Howard and Ruder, 2018), BERT (Devlin et al., 2018), to offer complementary performance gains, as has been shown for a few recent papers (Wang et al., 2018; Rajpurkar et al., 2018).

For claim veracity prediction without evidence documents with the MTL with LEL model, we use the following sentence encoding variants: claim- 
only, which uses a BiLSTM-based sentence embedding as input, and claim-only_embavg, which uses a sentence embedding based on mean averaged word embeddings as input.

We train one multi-task model per task (i.e., one model per domain). We perform a grid search over the following hyperparameters, tuned on the respective dev set, and evaluate on the correspoding test set (final settings are underlined): word embedding size [64, 128, 256], BiLSTM hidden layer size $[64, \underline{128}, 256]$, number of BiLSTM hidden layers $[1, \underline{2}, 3]$, BiLSTM dropout on input and output layers $[0.0, \underline{0.1}, 0.2,0.5]$, word-by-wordattention for BiLSTM with window size 10 (Bahdanau et al., 2014) [True, False], skip-connections for the BiLSTM [True, False], batch size [32, 64, 128], label embedding size [16, 32, 64]. We use ReLU as an activation function for both the BiLSTM and the CNN. For the CNN, the following hyperparameters are used: number filters [32], kernel size [32]. We train using cross-entropy loss and the RMSProp optimiser with initial learning rate of 0.001 and perform early stopping on the dev set with a patience of 3 .

\subsection{Results}

For each domain, we compute the Micro as well as Macro F1, then mean average results over all domains. Core results with all vs. no metadata are shown in Table 5. We first experiment with different base model variants and find that label embeddings improve results, and that the best proposed models utilising multiple domains outperform single-task models (see Table 8). This corroborates the findings of Augenstein et al. (2018). Per-domain results with the best model are shown in Table 6. Domain names are from hereon after abbreviated for brevity, see Table 11 in the appendix for correspondences to full website names. Unsurprisingly, it is hard to achieve a high Macro F1 for domains with many labels, e.g. tron and snes. Further, some domains, surprisingly mostly with small numbers of instances, seem to be very easy - a perfect Micro and Macro F1 score of 1.0 is achieved on ranz, bove, buca, fani and thal. We find that for those domains, the verdict is often already revealed as part of the claim using explicit wording.

Claim-Only vs. Evidence-Based Veracity Prediction. Our evidence-based claim veracity prediction models outperform claim-only veracity

\begin{tabular}{lrccc}
\hline Domain & \# Insts & \# Labs & Micro F1 & Macro F1 \\
\hline ranz & 21 & 2 & 1.000 & 1.000 \\
bove & 295 & 2 & 1.000 & 1.000 \\
abbc & 436 & 3 & 0.463 & 0.453 \\
huca & 34 & 3 & 1.000 & 1.000 \\
mpws & 47 & 3 & 0.667 & 0.583 \\
peck & 65 & 3 & 0.667 & 0.472 \\
faan & 111 & 3 & 0.682 & 0.679 \\
clck & 38 & 3 & 0.833 & 0.619 \\
fani & 20 & 3 & 1.000 & 1.000 \\
chct & 355 & 4 & 0.550 & 0.513 \\
obry & 59 & 4 & 0.417 & 0.268 \\
vees & 504 & 4 & 0.721 & 0.425 \\
faly & 111 & 5 & 0.278 & 0.5 \\
goop & 2943 & 6 & 0.822 & 0.387 \\
pose & 1361 & 6 & 0.438 & 0.328 \\
thet & 79 & 6 & 0.55 & 0.37 \\
thal & 163 & 7 & 1.000 & 1.000 \\
afck & 433 & 7 & 0.357 & 0.259 \\
hoer & 1310 & 7 & 0.694 & 0.549 \\
para & 222 & 7 & 0.375 & 0.311 \\
wast & 201 & 7 & 0.344 & 0.214 \\
vogo & 654 & 8 & 0.594 & 0.297 \\
pomt & 15390 & 9 & 0.321 & 0.276 \\
snes & 6455 & 12 & 0.551 & 0.097 \\
farg & 485 & 11 & 0.500 & 0.140 \\
tron & 3423 & 27 & 0.429 & 0.046 \\
\hline avg & & 7.17 & 0.625 & 0.492 \\
\hline & & & &
\end{tabular}

Table 6: Total number of instances and unique labels per domain, as well as per-domain results with model crawled_ranked + meta, sorted by label size

\begin{tabular}{lcc}
\hline Metadata & Micro F1 & Macro F1 \\
\hline None & $\mathbf{0 . 6 2 7}$ & 0.441 \\
\hline Speaker & 0.602 & 0.435 \\
+ Tags & 0.608 & 0.460 \\
\hline Tags & 0.585 & 0.461 \\
\hline Entity & 0.569 & 0.427 \\
+ Speaker & 0.607 & 0.477 \\
+ Tags & 0.625 & $\mathbf{0 . 4 9 2}$ \\
\hline
\end{tabular}

Table 7: Ablation results with base model crawled_ranked for different types of metadata

\begin{tabular}{lcc}
\hline Model & Micro F1 & Macro F1 \\
\hline STL & 0.527 & 0.388 \\
MTL & 0.556 & 0.448 \\
MTL + LEL & $\mathbf{0 . 6 2 5}$ & $\mathbf{0 . 4 9 2}$ \\
\hline
\end{tabular}

Table 8: Ablation results with crawled_ranked + meta encoding for STL vs. MTL vs. MTL + LEL training

prediction models by a large margin. Unsurprisingly, claim-only_embavg is outperformed by claim-only. Further, crawled_ranked is our bestperforming model in terms of Micro F1 and Macro $\mathrm{F} 1$, meaning that our model captures that not every piece of evidence is equally important, and can 


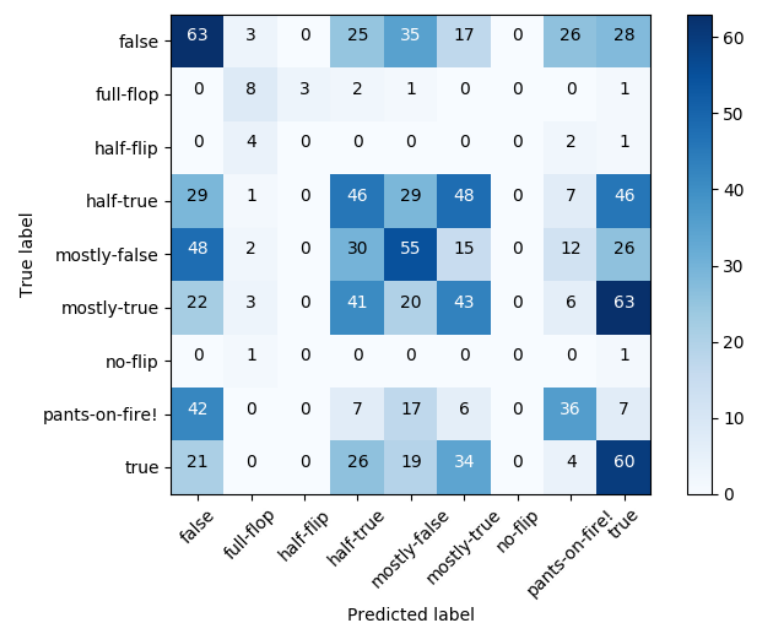

Figure 3: Confusion matrix of predicted labels with best-performing model, crawled_ranked + meta, on the 'pomt' domain

utilise this for veracity prediction.

Metadata. We perform an ablation analysis of how metadata impacts results, shown in Table 7. Out of the different types of metadata, topic tags on their own contribute the most. This is likely because they offer highly complementary information to the claim text of evidence pages. Only using all metadata together achieves a higher Macro F1 at similar Micro F1 than using no metadata at all. To further investigate this, we split the test set into those instances for which no metadata is available vs. those for which metadata is available. We find that encoding metadata within the model hurts performance for domains where no metadata is available, but improves performance where it is. In practice, an ensemble of both types of models would be sensible, as well as exploring more involved methods of encoding metadata.

\section{Analysis and Discussion}

An analysis of labels frequently confused with one another, for the largest domain 'pomt' and best-performing model crawled_ranked + meta is shown in Figure 3. The diagonal represents when gold and predicted labels match, and the numbers signify the number of test instances. One can observe that the model struggles more to detect claims with labels 'true' than those with label 'false'. Generally, many confusions occur over close labels, e.g. 'half-true' vs. 'mostly true'.

We further analyse what properties instances that are predicted correctly vs. incorrectly have, using the model crawled_ranked meta. We find that, unsurprisingly, longer claims are harder to classify correctly, and that claims with a high direct token overlap with evidence pages lead to a high evidence ranking. When it comes to frequently occurring tags and entities, very general tags such as 'government-and-politics' or 'tax' that do not give away much, frequently co-occur with incorrect predictions, whereas more specific tags such as 'brisbane-4000' or 'hong-kong' tend to co-occur with correct predictions. Similar trends are observed for bigrams. This means that the model has an easy time succeeding for instances where the claims are short, where specific topics tend to co-occur with certain veracities, and where evidence documents are highly informative. Instances with longer, more complex claims where evidence is ambiguous remain challenging.

\section{Conclusions}

We present a new, real-world fact checking dataset, currently the largest of its kind. It consists of 34,918 claims collected from 26 fact checking websites, rich metadata and 10 retrieved evidence pages per claim. We find that encoding the metadata as well evidence pages helps, and introduce a new joint model for ranking evidence pages and predicting veracity.

\section{Acknowledgments}

This research is partially supported by QUARTZ (721321, EU H2020 MSCA-ITN) and DABAI (5153-00004A, Innovation Fund Denmark).

\section{References}

Isabelle Augenstein, Tim Rocktäschel, Andreas Vlachos, and Kalina Bontcheva. 2016a. Stance Detection with Bidirectional Conditional Encoding. In Proceedings of the 2016 Conference on Empirical Methods in Natural Language Processing, pages 876-885, Austin, Texas. Association for Computational Linguistics.

Isabelle Augenstein, Sebastian Ruder, and Anders Søgaard. 2018. Multi-Task Learning of Pairwise Sequence Classification Tasks over Disparate Label Spaces. In NAACL-HLT, pages 1896-1906. Association for Computational Linguistics.

Isabelle Augenstein, Andreas Vlachos, and Kalina Bontcheva. 2016b. USFD at SemEval-2016 Task 6: Any-Target Stance Detection on Twitter with Autoencoders. In Proceedings of the 10th International Workshop on Semantic Evaluation (SemEval-2016), pages 389-393, San Diego, California. Association for Computational Linguistics. 
Joan Bachenko, Eileen Fitzpatrick, and Michael Schonwetter. 2008. Verification and implementation of language-based deception indicators in civil and criminal narratives. In Proceedings of the 22nd International Conference on Computational Linguistics-Volume 1, pages 41-48. Association for Computational Linguistics.

Dzmitry Bahdanau, Kyunghyun Cho, and Yoshua Bengio. 2014. Neural Machine Translation by Jointly Learning to Align and Translate. In Proceedings of ICLR.

Ramy Baly, Mitra Mohtarami, James R. Glass, Lluís Màrquez, Alessandro Moschitti, and Preslav Nakov. 2018. Integrating Stance Detection and Fact Checking in a Unified Corpus. In Proceedings of the 2018 Conference of the North American Chapter of the Association for Computational Linguistics: $\mathrm{Hu}$ man Language Technologies, NAACL-HLT, New Orleans, Louisiana, USA, June 1-6, 2018, Volume 2 (Short Papers), pages 21-27. Association for Computational Linguistics.

Alberto Barrn-Cedeo, Tamer Elsayed, Reem Suwaileh, Llus Mrquez, Pepa Atanasova, Wajdi Zaghouani, Spas Kyuchukov, Giovanni Da San Martino, and Preslav Nakov. 2018. Overview of the CLEF-2018 CheckThat! Lab on automatic identification and verification of political claims. Task 2: Factuality. In CLEF (Working Notes), volume 2125 of CEUR Workshop Proceedings. CEUR-WS.org.

Rich Caruana. 1993. Multitask Learning: A Knowledge-Based Source of Inductive Bias. In Proceedings of ICML.

Sihao Chen, Daniel Khashabi, Wenpeng Yin, Chris Callison-Burch, and Dan Roth. 2019. Seeing Things from a Different Angle: Discovering Diverse Perspectives about Claims. In Proceedings of NAACL.

G L Ciampaglia, P Shiralkar, L M Rocha, J Bollen, F Menczer, and A Flammini. 2015. Computational Fact Checking from Knowledge Networks. PLoS One, 10(6).

Leon Derczynski, Kalina Bontcheva, Maria Liakata, Rob Procter, Geraldine Wong Sak Hoi, and Arkaitz Zubiaga. 2017. SemEval-2017 Task 8: RumourEval: Determining rumour veracity and support for rumours. In Proceedings of the 11th International Workshop on Semantic Evaluation (SemEval-2017), pages 69-76. Association for Computational Linguistics.

Jacob Devlin, Ming-Wei Chang, Kenton Lee, and Kristina Toutanova. 2018. BERT: Pre-training of Deep Bidirectional Transformers for Language Understanding. CoRR, abs/1810.04805.

Omar Enayet and Samhaa R. El-Beltagy. 2017. NileTMRG at SemEval-2017 Task 8: Determining Rumour and Veracity Support for Rumours on Twitter. In Proceedings of the 11th International
Workshop on Semantic Evaluation (SemEval-2017), pages 470-474. Association for Computational Linguistics.

William Ferreira and Andreas Vlachos. 2016. Emergent: a novel data-set for stance classification. In HLT-NAACL, pages 1163-1168. The Association for Computational Linguistics.

Jeremy Howard and Sebastian Ruder. 2018. Universal Language Model Fine-tuning for Text Classification. In ACL (1), pages 328-339. Association for Computational Linguistics.

Georgi Karadzhov, Pepa Gencheva, Preslav Nakov, and Ivan Koychev. 2017. We Built a Fake News / Click Bait Filter: What Happened Next Will Blow Your Mind! In RANLP 2017, pages 334-343.

Elena Kochkina, Maria Liakata, and Isabelle Augenstein. 2017. Turing at SemEval-2017 Task 8: Sequential Approach to Rumour Stance Classification with Branch-LSTM. In Proceedings of the 11th International Workshop on Semantic Evaluation (SemEval-2017), pages 475-480, Vancouver, Canada. Association for Computational Linguistics.

Nikolaos Kolitsas, Octavian-Eugen Ganea, and Thomas Hofmann. 2018. End-to-End Neural Entity Linking. In Proceedings of the 22nd Conference on Computational Natural Language Learning, pages 519-529. Association for Computational Linguistics.

Rada Mihalcea and Carlo Strapparava. 2009. The lie detector: Explorations in the automatic recognition of deceptive language. In Proceedings of the ACLIJCNLP 2009 Conference Short Papers, pages 309312. Association for Computational Linguistics.

Tsvetomila Mihaylova, Preslav Nakov, Lluís Màrquez, Alberto Barrón-Cedeño, Mitra Mohtarami, Georgi Karadzhov, and James R. Glass. 2018. Fact Checking in Community Forums. In Proceedings of the Thirty-Second AAAI Conference on Artificial Intelligence, New Orleans, Louisiana, USA, February 2-7, 2018. AAAI Press.

Tanushree Mitra and Eric Gilbert. 2015. Credbank: A large-scale social media corpus with associated credibility annotations. In ICWSM, pages 258-267.

Lili Mou, Rui Men, Ge Li, Yan Xu, Lu Zhang, Rui Yan, and Zhi Jin. 2016. Natural Language Inference by Tree-Based Convolution and Heuristic Matching. In $A C L$ (2). The Association for Computer Linguistics.

Verónica Pérez-Rosas, Bennett Kleinberg, Alexandra Lefevre, and Rada Mihalcea. 2018. Automatic Detection of Fake News. In Proceedings of the 27th International Conference on Computational Linguistics, pages 3391-3401. Association for Computational Linguistics. 
Matthew E. Peters, Mark Neumann, Mohit Iyyer, Matt Gardner, Christopher Clark, Kenton Lee, and Luke Zettlemoyer. 2018. Deep Contextualized Word Representations. In NAACL-HLT, pages 2227-2237. Association for Computational Linguistics.

Dean Pomerleau and Delip Rao. 2017. The Fake News Challenge: Exploring how artificial intelligence technologies could be leveraged to combat fake news. http://www. fakenewschallenge. org/. Accessed: 2019-02-14.

Kashyap Popat, Subhabrata Mukherjee, Jannik Strötgen, and Gerhard Weikum. 2016. Credibility Assessment of Textual Claims on the Web. In CIKM, pages 2173-2178.

Pranav Rajpurkar, Robin Jia, and Percy Liang. 2018. Know What You Don't Know: Unanswerable Questions for SQuAD. In ACL (2), pages 784-789. Association for Computational Linguistics.

Benjamin Riedel, Isabelle Augenstein, Georgios P. Spithourakis, and Sebastian Riedel. 2017. A simple but tough-to-beat baseline for the Fake News Challenge stance detection task. CoRR, abs/1707.03264.

Victoria Rubin, Niall Conroy, Yimin Chen, and Sarah Cornwell. 2016. Fake News or Truth? Using Satirical Cues to Detect Potentially Misleading News. In Proceedings of the Second Workshop on Computational Approaches to Deception Detection, pages 717. Association for Computational Linguistics.

K. Shu, D. Mahudeswaran, S. Wang, D. Lee, and H. Liu. 2018. FakeNewsNet: A Data Repository with News Content, Social Context and Dynamic Information for Studying Fake News on Social Media. ArXiv e-prints.

Eugenio Tacchini, Gabriele Ballarin, Marco L. Della Vedova, Stefano Moret, and Luca de Alfaro. 2017. Some Like it Hoax: Automated Fake News Detection in Social Networks. In Proceedings of the Second Workshop on Data Science for Social Good (SoGood), volume 1960 of CEUR Workshop Proceedings.

James Thorne, Andreas Vlachos, Christos Christodoulopoulos, and Arpit Mittal. 2018. FEVER: a Large-scale Dataset for Fact Extraction and VERification. In Proceedings of the 2018 Conference of the North American Chapter of the Association for Computational Linguistics: Human Language Technologies, Volume 1 (Long Papers), pages 809-819, New Orleans, Louisiana. Association for Computational Linguistics.

Andreas Vlachos and Sebastian Riedel. 2014. Fact Checking: Task definition and dataset construction. In Proceedings of the ACL 2014 Workshop on Language Technologies and Computational Social Science, pages 18-22. Association for Computational Linguistics.
Soroush Vosoughi, Deb Roy, and Sinan Aral. 2018. The spread of true and false news online. Science, 359(6380):1146-1151.

Alex Wang, Amanpreet Singh, Julian Michael, Felix Hill, Omer Levy, and Samuel R. Bowman. 2018. GLUE: A Multi-Task Benchmark and Analysis Platform for Natural Language Understanding. In BlackboxNLP@EMNLP, pages 353-355. Association for Computational Linguistics.

William Yang Wang. 2017. "Liar, Liar Pants on Fire": A New Benchmark Dataset for Fake News Detection. In Proceedings of the 55th Annual Meeting of the Association for Computational Linguistics (Volume 2: Short Papers), pages 422-426. Association for Computational Linguistics.

Wenpeng Yin and Dan Roth. 2018. TwoWingOS: A Two-Wing Optimization Strategy for Evidential Claim Verification. In Proceedings of the 2018 Conference on Empirical Methods in Natural Language Processing, pages 105-114, Brussels, Belgium. Association for Computational Linguistics.

Arkaitz Zubiaga, Elena Kochkina, Maria Liakata, Rob Procter, Michal Lukasik, Kalina Bontcheva, Trevor Cohn, and Isabelle Augenstein. 2018. Discourseaware rumour stance classification in social media using sequential classifiers. Informatino Processing \& Management, 54(2):273-290.

Arkaitz Zubiaga, Maria Liakata, Rob Procter, Geraldine Wong Sak Hoi, and Peter Tolmie. 2016. Analysing how people orient to and spread rumours in social media by looking at conversational threads. PLOS ONE, 11(3):1-29. 


\begin{tabular}{ll}
\hline Websites (Sources) & Reason \\
\hline Mediabiasfactcheck & Website that checks other news websites \\
CBC & No pattern to crawl \\
apnews.com/APFactCheck & No categorical label and no structured claim \\
weeklystandard.com/tag/fact-check & Mostly no label, and they are placed anywhere \\
ballotpedia.org & No categorical label and no structured claim \\
channel3000.com/news/politics/reality-check & No categorical label, lack of structure, and no clear claim \\
npr.org/sections/politics-fact-check & No label and no clear claim (only some titles are claims) \\
dailycaller.com/buzz/check-your-fact & Is a subset of checkyourfact which has already been crawled \\
sacbee.com & Contains very few labelled articles, and without clear claims \\
TheGuardian & Only a few websites have a pattern for labels. \\
\hline
\end{tabular}

Table 9: The list of websites that we did not crawl and reasons for not crawling them.

\begin{tabular}{|c|c|c|c|}
\hline \multicolumn{2}{|c|}{ Domain \# Insts } & \multirow{2}{*}{ \# Labels } & \multirow{2}{*}{$\begin{array}{l}\text { Labels } \\
\text { in-between, in-the-red, in-the-green }\end{array}$} \\
\hline abbc & 436 & & \\
\hline afck & 433 & 7 & $\begin{array}{l}\text { correct, incorrect, mostly-correct, unproven, misleading, understated, exagger- } \\
\text { ated }\end{array}$ \\
\hline bove & 295 & 2 & none, rating: false \\
\hline chet & 355 & 4 & verdict: true, verdict: false, verdict: unsubstantiated, none \\
\hline clck & 38 & 3 & incorrect, unsupported, misleading \\
\hline faan & 111 & 3 & factscan score: false, factscan score: true, factscan score: misleading \\
\hline faly & 71 & 5 & true, none, partly true, unverified, false \\
\hline fani & 20 & 3 & conclusion: accurate, conclusion: false, conclusion: unclear \\
\hline farg & 485 & 11 & $\begin{array}{l}\text { false, none, distorts the facts, misleading, spins the facts, no evidence, not the } \\
\text { whole story, unsupported, cherry picks, exaggerates, out of context }\end{array}$ \\
\hline goop & 2943 & 6 & $0,1,2,3,4,10$ \\
\hline hoer & 1310 & 7 & $\begin{array}{l}\text { facebook scams, true messages, bogus warning, statirical reports, fake news, } \\
\text { unsubstantiated messages, misleading recommendations }\end{array}$ \\
\hline huca & 34 & 3 & a lot of baloney, a little baloney, some baloney \\
\hline mpws & 47 & 3 & accurate, false, misleading \\
\hline obry & 59 & 4 & mostly_true, verified, unobservable, mostly_false \\
\hline para & 222 & 7 & mostly false, mostly true, half-true, false, true, pants on fire!, half flip \\
\hline peck & 65 & 3 & false, true, partially true \\
\hline pomt & 15390 & 9 & $\begin{array}{l}\text { half-true, false, mostly true, mostly false, true, pants on fire!, full flop, half flip, } \\
\text { no flip }\end{array}$ \\
\hline pose & 1361 & 6 & promise kept, promise broken, compromise, in the works, not yet rated, stalled \\
\hline $\operatorname{ranz}$ & & 2 & fact, fiction . \\
\hline snes & 6455 & 12 & $\begin{array}{l}\text { false, true, mixture, unproven, mostly false, mostly true, miscaptioned, legend, } \\
\text { outdated, misattributed, scam, correct attribution }\end{array}$ \\
\hline thet & 79 & 6 & none, mostly false, mostly true, half true, false, true \\
\hline thal & 74 & 2 & none, we rate this claim false \\
\hline tron & 3423 & 27 & $\begin{array}{l}\text { fiction!, truth!, unproven!, truth! \& fiction!, mostly fiction!, none, disputed!, } \\
\text { truth! \& misleading!, authorship confirmed!, mostly truth!, incorrect attribu- } \\
\text { tion!, scam!, investigation pending!, confirmed authorship!, commentary!, pre- } \\
\text { viously truth! now resolved!, outdated!, truth! \& outdated!, virus!, fiction! \& } \\
\text { satire!, truth! \& unproven!, misleading!, grass roots movement!, opinion!, cor- } \\
\text { rect attribution!, truth! \& disputed!, inaccurate attribution! }\end{array}$ \\
\hline vees & 504 & 4 & none, fake, misleading, false \\
\hline vogo & 653 & 8 & $\begin{array}{l}\text { none, determination: false, determination: true, determination: mostly true, } \\
\text { determination: misleading, determination: barely true, determination: huckster } \\
\text { propaganda, determination: false, determination: a stretch }\end{array}$ \\
\hline wast & 201 & 7 & $\begin{array}{l}4 \text { pinnochios, } 3 \text { pinnochios, } 2 \text { pinnochios, false, not the whole story, needs } \\
\text { context, none }\end{array}$ \\
\hline
\end{tabular}

Table 10: Number of instances, and labels per domain sorted by number of occurrences 


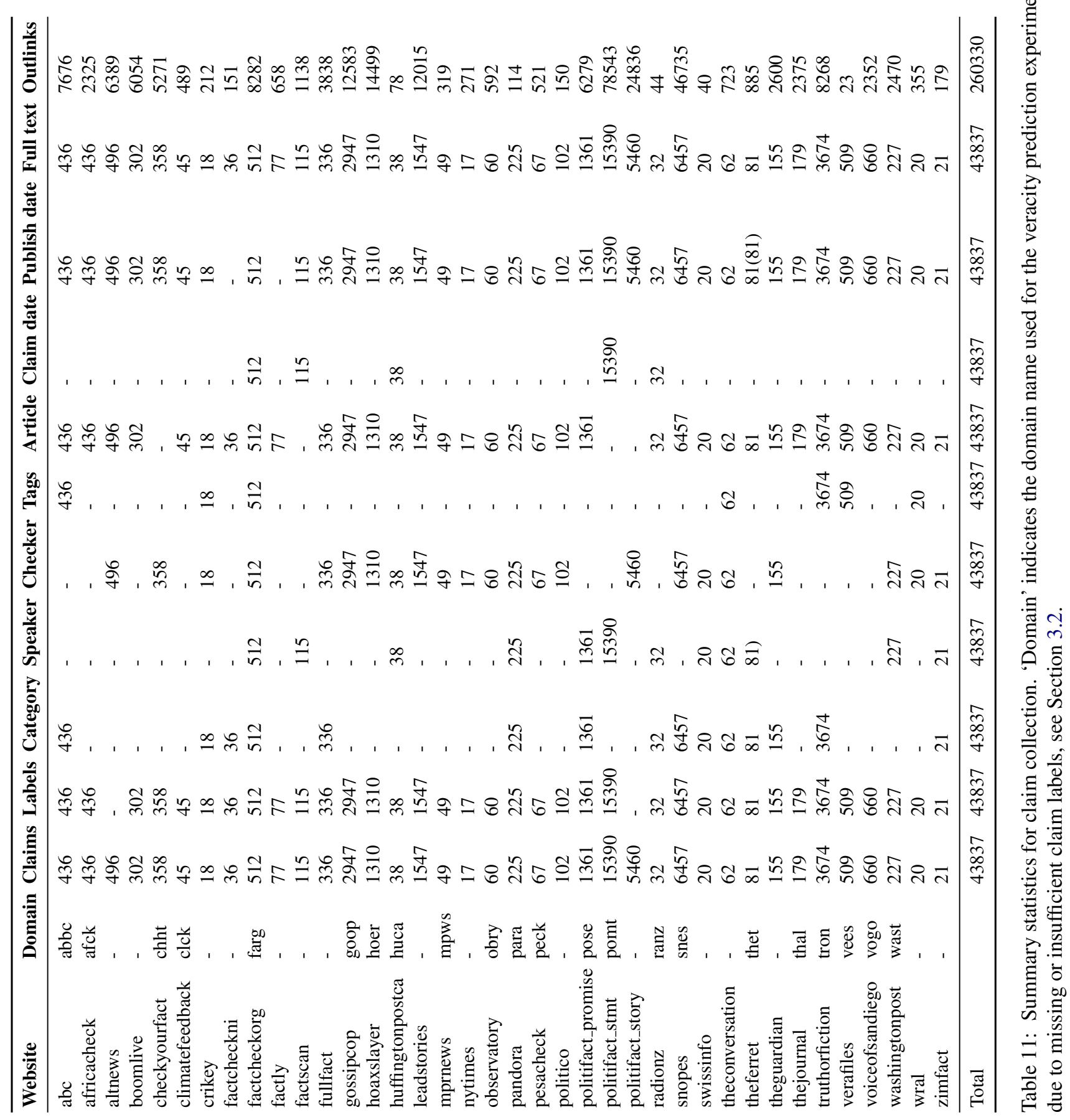

